1 - ORIGINAL ARTICLE ISCHEMIA-REPERFUSION

\title{
Ischemic preconditioning modifies mortality and inflammatory response ${ }^{1}$
}

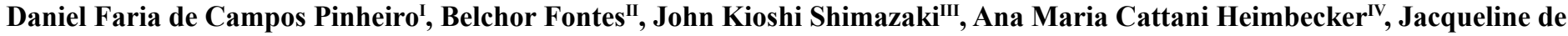 \\ Fátima Jacysynv, Samir Rasslan ${ }^{\text {VI Edna Frasson de Souza Montero }{ }^{\text {VII }} \text {, Edivaldo Massazo Utiyama }}$ VIII
}

DOI: http://dx.doi.org/10.1590/S0102-865020160010000001

IFellow PhD degree, Postgraduate Program in Surgical Clinics, General and Trauma Surgery Division, Department of Surgery, Medical School, Universidade de São Paulo (FMUSP), Brazil. Design of the study, surgical procedures, statistical analysis, interpretation of data, manuscript writing. IIPhD, General and Trauma Surgery Division, Department of Surgery, Medical School, FMUSP, Sao Paulo-SP, Brazil. Design of the study, statistical analysis, interpretation of data, manuscript writing.

IIIGraduate student, FMUSP. Grant from Institutional Program for Scientific Initiation (PIBIC) of the National Council of Scientific and Technological Development (CNPq) Ministry of Science, Technology and Innovation, Brazil. Surgical procedures.

${ }^{\mathrm{IV}}$ Biologist, Laboratory of Surgical Physiopathology (LIM-62), Department of Surgery, FMUSP, Brazil. Experimental procedures.

${ }^{\mathrm{v}} \mathrm{PhD}$, Biologist, Laboratory of Surgical Physiopathology (LIM-62), Department of Surgery, FMUSP, Sao Paulo-SP, Brazil. Inflammatory and oxidative stress mediators' analysis.

${ }^{\mathrm{V}}$ Full Professor, General and Trauma Surgery Division, Department of Surgery, FMUSP, Sao Paulo-SP, Brazil. Interpretation of data, manuscript writing.

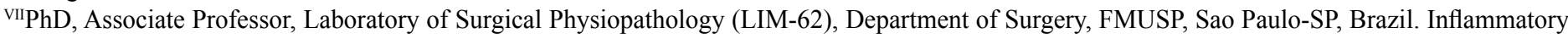
and oxidative stress mediators' analysis, interpretation of data, critical revision.

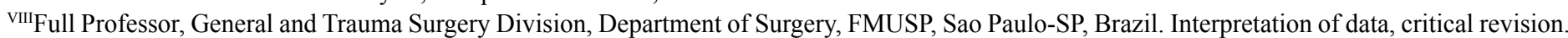

\section{ABSTRACT}

PURPOSE: To evaluate the effect of ischemic preconditioning on mortality, inflammatory mediators and oxidative stress after intestinal ischemia and reperfusion.

METHODS: Male Wistar rats were allocated according to the period of ischemia with or without ischemic preconditioning which consist on clamping the superior mesenteric artery for 10 minutes followed by reperfusion for 10 minutes before the sustained ischemia period. Mortality was assessed in Phase 1 study, and the CINC-1, CINC-2 and MDA levels in the lungs were analyzed in Phase 2.

RESULTS: Mortality was lower in the ischemic preconditioning group subjected to 90 minutes of ischemia compared to the group without ischemic preconditioning (I-90: 50\% and IPC-90: 15\%, $\mathrm{p}=0.018$ ), and it was lower in the ischemic preconditioning group as a whole compared to the groups without ischemic preconditioning (IPC-14\% and I=30\%, p=0.006). Lower levels of MDA, CINC-1, and CINC-2 were observed in the animals that were subjected to ischemic preconditioning compared to the animals that were not (MDA: I-45=1.23 nmol $/ \mathrm{mg}$ protein, and IPC-45 $=0.62 \mathrm{nmol} / \mathrm{mg}$ protein, $\mathrm{p}=0.0333 ; \mathrm{CINC}-1: \mathrm{I}-45=0.82 \mathrm{ng} / \mathrm{mL} \mathrm{and} \mathrm{IPC}-45=0.67 \mathrm{ng} / \mathrm{mL}$, $\mathrm{p}=0.041$; CINC-2: $\mathrm{I}-45=0.52 \mathrm{ng} / \mathrm{mL}$ and IPC $-45=0.35 \mathrm{ng} / \mathrm{mL}, \mathrm{p}=0.032$ ).

CONCLUSION: Ischemic preconditioning reduces mortality, inflammatory process and oxidative stress in rats subjected to intestinal ischemia and reperfusion.

Key words: Ischemic Preconditioning. Reperfusion. Mortality. Oxidative Stress. Rats. 


\section{Introduction}

Intestinal ischemia/reperfusion (I/R) resulting from the superior mesenteric artery (SMA) occlusion can lead to intestinal mucosal injury which in turn releases inflammatory mediators, resulting in a systemic inflammatory response. The consequence may be multiple organ damage, especially acute lung injury that may culminate into multiple organs dysfunction and failure ${ }^{1-4}$. The mechanism underlying these processes is highly complex and involves the action and interaction of cellular mediators, such as macrophages, neutrophils and endothelium, as well as humoral molecules, such as lipopolysaccharide, superoxide radicals and cytokines. Lipid peroxidation also occurs and there is an increase of malondialdehyde levels, leukocyte-endothelial adhesion, accumulation of polymorphonuclear neutrophils in the lungs alveoli, and polymorphonuclear neutrophils apoptosis ${ }^{4,5}$. Interleukin-8 (and its homologous CINC-1 and CINC-2 in rats) plays an important role in the pathogenesis of inflammatory bowel disease induced by intestinal $\mathrm{I} / \mathrm{R}^{5-7}$. Among the different models of intestinal $\mathrm{I} / \mathrm{R}$ in rats, the model involving 45 minutes of ischemia has been considered to be free of mortality ${ }^{7-10}$. The models having ischemia periods longer than 45 minutes have varying mortality rates that have not been clearly determined for these ischemia periods $^{10,11}$.

Intestinal preconditioning, compared to hypotermia and modulation of inflammatory mediators ${ }^{11}$, IPC, first described by Murry et al. ${ }^{12}$, consists of one or multiple short periods of ischemia followed by the same periods of reperfusion, applied prior to prolonged or appropriate ischemia ${ }^{13-16}$ was found to be the most promising strategy to improve intestinal tolerance to I/R injury, and to protect other organs such as lungs, liver, brain, kidneys, pancreas by reducing I/R damage ${ }^{16-19}$.

Hotter et $a l .{ }^{13}$ described the first rat model of IPC preceding 90-min of SMA occlusion. Others reported beneficial effects of IPC in intestinal transplantation ${ }^{14,19,20}$. The attenuation of the increased permeability of the mucosa by adenosine deaminase in an intestinal $\mathrm{I} / \mathrm{R}$ model suggested that adenosine may be a mediator of $\mathrm{IPC}^{18}$. Overall, the studies of the mechanism of IPC in animal models of intestinal I/R have shown that the protective effect of IPC is attributable to the production of various inflammatory mediators ${ }^{20-22}$. Thus, IPC can be considered a potentially promising strategy to minimize reperfusion injury, and further studies are needed to clarify this strategy ${ }^{22,23}$.

The effect of IPC on mortality in rats subjected to intestinal $\mathrm{I} / \mathrm{R}$ has been described in the literature, but with inconclusive results $^{9,10}$ and the mortality rates after various periods intestinal
I/R have not been clearly established. Moreover, although IPC has been shown to decrease the severity of intestinal $I / R$ injuries, its effect on the mortality of the animals in these experiments has not been clearly determined.

The aim of this study was to determine the mortality rates caused by different periods of intestinal I/ $\mathrm{R}$ in rats, and to evaluate the effect of IPC on these mortality rates, and also to evaluate the effect of IPC on the levels of the inflammatory mediators and on the marker of oxidative stress.

\section{Methods}

This research project was approved by the Ethics Committee for animal care and use for experimental research of the Faculty of Medicine, University of São Paulo (FMUSP), and was conducted at the Laboratory of Surgical Physiopathology (LIM-62) Department of Surgery.

It was used 254 Wistar male rats from the Central Animal Laboratory of the FMUSP. The study was divided into Phase 1 (mortality) and Phase 2 (inflammatory and oxidative stress mediators). The animals were weighed and then anesthetized with an intraperitoneal injection of ketamine hydrochloride 5\% (Ketalar $^{\circledR}$, Cristalia) and xylazine 2\% (Rompum $^{\circledR}$, Bayer). A median laparotomy was performed, and the superior mesenteric artery (SMA) was exposed at its origin.

Phase 1-Mortality. The animals were allocated into the following 11 groups.

Group 1 ( $n=30)$ - control group; the animals underwent anesthesia and a median laparotomy with identification and isolation of the SMA without inducing ischemia; Group $2(n=26)$ - 45 minutes of mesenteric ischemia (I-45); the animals underwent 45 minutes of mesenteric ischemia by means of clamping the SMA at its origin;

Group $3(n=20)-60$ minutes of mesenteric ischemia (I-60);

Group $4(n=20)-75$ minutes of mesenteric ischemia

Group $5(\mathrm{n}=26)$ - 90 minutes of mesenteric ischemia (I-90); (I-105);

Group $6(n=20)-105$ minutes of mesenteric ischemia

Group $7(n=26)$ - IPC +45 minutes of mesenteric ischemia (IPC-45); the animals were subjected to IPC by SMA occlusion for 10 minutes followed by reperfusion for 10 minutes once and then 45 minutes of mesenteric ischemia;

Group $8(n=20)-$ IPC +60 minutes of mesenteric ischemia (IPC-60); 
Group $9(\mathrm{n}=20)-$ IPC +75 minutes of mesenteric ischemia (IPC-75);

Group $10(n=20)$ - IPC +90 minutes of mesenteric ischemia (IPC-90);

Group $11(n=20)$ - IPC +105 minutes of mesenteric ischemia (IPC-105).

After the sustained ischemia, the animals had its abdominal cavity closed and were kept under observation for seven days with free access to water and food to observe their mortality.

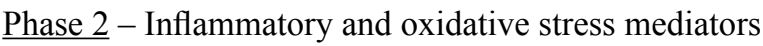

The evaluation of the changes in the inflammatory mediators and oxidative stress marker was performed in the following four groups: Group 2 (I-45), Group 5 (I-90), Group 7 (IPC-45) and Group 10 (IPC-90). These groups were selected after the statistical analysis of the results of the Phase 1 study. The aim of the Phase 2 study was to evaluate the changes in the inflammatory mediators as a function of the variation of the ischemia duration, either in the presence and absence of IPC. A total of 24 animals, 6 from each group (Groups 2, 5, 7 and 10), were employed in the Phase 2 study.

The difference between the initial procedures for the Phase 2 study, compared to the Phase 1 study, was the time of reperfusion that was established as 2 hours for the Phase 2 study. At this point, rats were anesthetized again for reoperation, and both lungs were removed for analysis. The rats were sacrificed by exsanguination under anesthesia.

\section{Inflammatory mediators (CINC-1 and CINC-2)}

The levels of the systemic inflammatory mediators, CINC-1 and CINC-2, in the lungs were determined by using ELISA (Enzyme-linked immunosorbent assay method), using commercially available kits and following the manufacturer's instructions (R \& D Systems Inc., Minneapolis, MN, USA).

\section{Malondialdehyde}

The quantification of malondialdehyde in the lungs was performed using the thiobarbituric acid reactive substances technique as described elsewhere ${ }^{24}$. It is based on a reaction with thiobarbituric acid in low $\mathrm{pH}$ and high temperature forming the MDA-TBA (malondialdehyde-thiobarbituric acid) complex, which presents characteristic color and absorption. Those characteristics can be detected through spectrophotometry.

\section{Statistical analysis}

For the statistical analysis, the results were organized into tables and graphs. The variables, including initial weight, final weight, and weight gain, were subjected to the KolmogorovSmirnov test to assess the normality of the distribution. The initial weight was normally distributed among the 11 groups in the study. Then, we tested whether this variable was uniformly distributed among the various groups using an analysis of variance with a criterion (One-way ANOVA).

Because the weight gain was not normally distributed among the 11 groups in the study, we applied the nonparametric Mann-Whitney test. To evaluate the differences between the groups with IPC and the groups without IPC, all of the IPC groups and the ischemia without IPC groups were matched according to the duration of ischemia $(45,60,75,90$ and 105 minutes), and then, these groups were compared with the control group.

The analysis of outcome (survival or death) was performed using the measures of association (chi-square test) in the following groups: IPC groups versus ischemia without IPC groups (45, 60, 75, 90 and 105 minutes), i.e., the IPC groups (IPC-45, IPC-60, IPC-75, IPC-90 and IPC-105) versus the ischemia without IPC groups (I-45, I-60, I-75, I-90 and I-105) and the control group.

For the qualitative variables (CINC-1, CINC-3 and MDA), we analyzed the median absolute $(\mathrm{N})$ and relative frequencies (\%). The results of the measurements of the inflammatory mediators (CINC-1 and CINC-2), as well as those of the marker of oxidative stress (malondialdehyde), which were considered as parametric variables, were evaluated using the ANOVA test. In cases with a significant difference, the Tukey test was used to analyze the pairs. The Graph Pad Prism version 6.0 program was used for statistical analysis, and the significance level was $5 \%$ for all of the tests.

\section{Results}

\section{Mortality - Phase 1}

In this phase, 230 animals were included: 20 in each of groups 2 to 11, and 30 animals in the control group (CTR). The mortality rates observed in the groups are presented in Figure 1. The mortality rate increased as the duration of ischemia increased, and no mortality was observed in the control group (CTR) or in the I-45 and IPC-45 groups (Figure 1). A significant difference was observed between the I-90 and IPC-90 groups $(\mathrm{p}=0.018)$. The lower mortality observed in the IPC groups with 60,75 or 105 
minutes of ischemia was not significant. However, the mortality rates observed in all groups with IPC, when taken together, were significantly small when compared with the similar rates of the groups without IPC, also taken together (Figure 2).

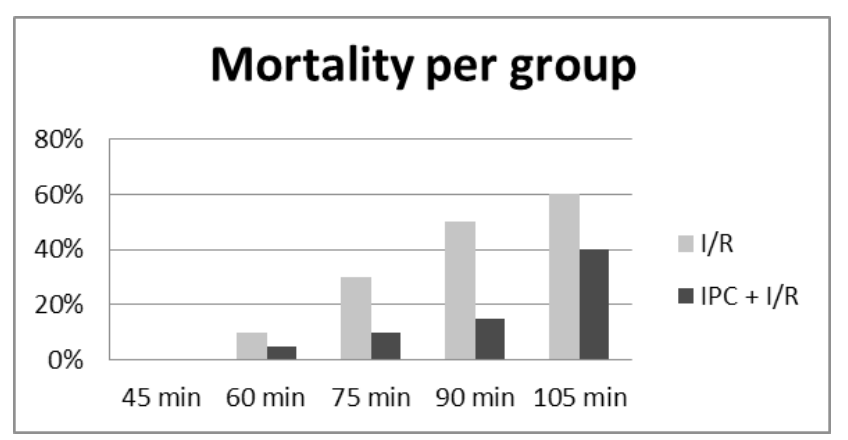

FIGURE 1 - Comparison of the mortality observed in the groups subjected to $I / R$ with those subjected to IPC $+I / R$ in the diverse duration of $I / R$.

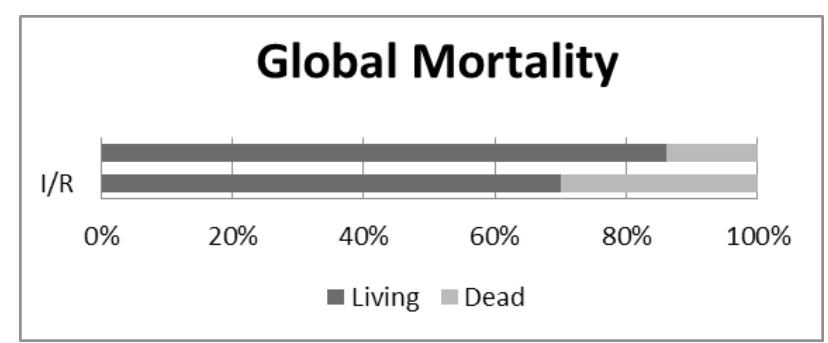

FIGURE 2 - Compares the mortality observed when all the groups undergoing IPC, taken together, were compared with those undergoing only I/R.

\section{Phase 2 - Evaluation of inflammatory mediators}

We observed a significant increase in both CINC1 and CINC-2 levels in IPC-45 compared to I-45 groups. The malondialdehyde levels in the lungs were significantly decreased in IPC-45 compared with I-45 groups. No significant difference was observed in any of the measurements (CINC-1, CINC-2 and malondialdehyde levels) between the I-90 and IPC-90 groups (Figures 3 to 4 ).

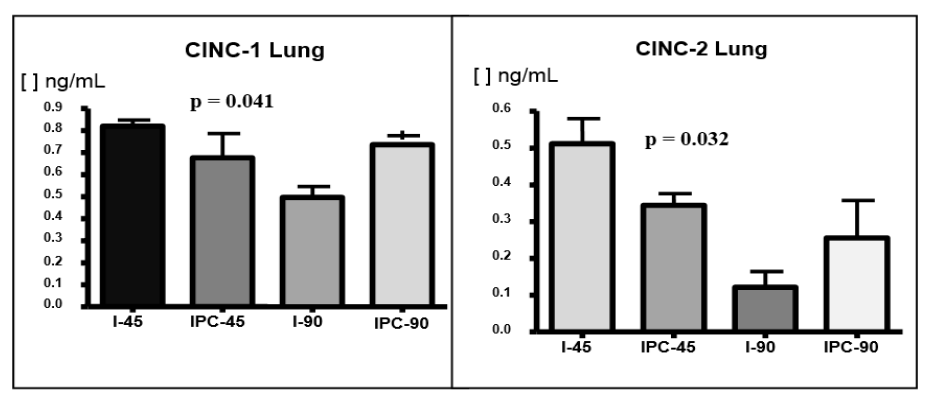

FIGURE 3 - CINC-1 and CINC-2 values in the lung according to the groups.

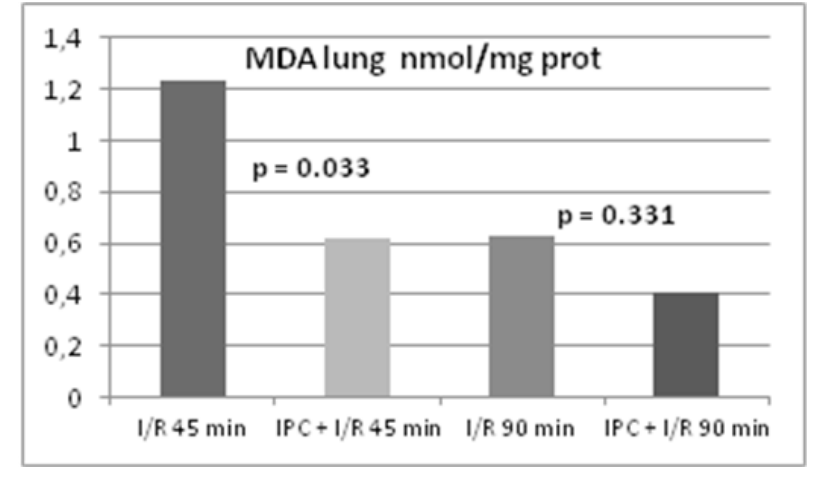

FIGURE 4 - The malondialdehyde (MDA) levels in the lung (groups 45 minutes and 90 minutes of ischemia, followed by 2 hours of reperfusion, with or without ischemic preconditioning (IPC)) in Phase 2. I/R -ischemia and reperfusion.

\section{Discussion}

The study of intestinal ischemia/reperfusion $(\mathrm{I} / \mathrm{R})$ is of great importance because the deleterious effects of $I / R$ may aggravate the clinical conditions of patients undergoing major surgery (i.e., cardiac, vascular, transplant, strangulated hernias and neonatal necrotizing enterocolitis) and also of patients in certain emergency medical situations, such as severe trauma, extensive burns, hemorrhagic shock and septic shock. Furthermore, in cases of prolonged ischemia, intestinal I/R may cause distant organ damage due to a systemic inflammatory response syndrome ${ }^{22,23}$. Because the intestine is the most perfused organ, it is usually affected by I/R during major cardiac surgery, myocardial infarction and vascular surgery ${ }^{25}$, and it is likely the most sensitive to $\mathrm{I} / \mathrm{R}$ injury ${ }^{11,26}$. Although intestinal ischemia is uncommon in heart surgeries $(0.53 \%$ to $3.7 \%)$; when present, it is associated with high mortality ( $64 \%$ to $100 \%$ of the cases $)^{27}$.

Kubes et $a l .{ }^{28}$ showed that most of the liver I/R models (including liver transplantation) have some degree of intestinal ischemia, which enhances the liver injury by inducing the release of toxins and intestinal neutrophil recruitment ${ }^{27,29}$. Recently, intestinal transplantation has become the preferred therapy for patients with short bowel syndrome, with intolerance to total parenteral nutrition ${ }^{14,17,19}$. However, the lesion caused by intestinal $\mathrm{I} / \mathrm{R}$ is a limiting factor for successful intestinal transplantation ${ }^{19}$, and it is as important as the proper preservation of the intestinal graft for minimizing the I/R injury ${ }^{14,17}$.

Since even short periods of mesenteric ischemia can damage the intestinal mucosa, then intestinal I/R can cause major changes in the mucosal barrier, leading to translocation and release of endotoxins in the bloodstream ${ }^{22,23}$, causing systemic inflammatory response and $\mathrm{MOSF}^{2}$. Therefore, controlling 
intestinal $\mathrm{I} / \mathrm{R}$ injury is important to achieve successful intestinal transplantation ${ }^{14,17,22}$. Among the several strategies developed to minimize the deleterious effects of intestinal I/R, IPC has great importance because it protects the intestine against I/R injury ${ }^{20}$.

The 10 minutes IPC was chose for use in this study because it has been demonstrated to protect organs against $I / R$ injury $^{3,11,14,19,30}$. Based on the statistical analysis of the results of the Phase 1 of this study, we chose the periods of 45 and 90 minutes of ischemia for the Phase 2 because 45 minutes was confirmed as free from mortality, either with or without IPC, and 90 minutes caused significantly less mortality when IPC was applied. Based on the literature supporting that acute intestinal and acute lungs injuries are detectable at two hours after 45 minutes of intestinal ischemia ${ }^{2}$, we chose two hours of reperfusion to evaluate the effect of IPC on the levels of the inflammatory mediators CINC-1 and CINC-2, and the marker of oxidative stress malondialdehyde.

Phase 1 of this study showed increased mortality rates as the duration of ischemia increased. This was expected once longer ischemia periods lead to greater organ damage, with increased injury degrees, with greater chances of occurring systemic inflammatory response, MOSF and death ${ }^{23}$. In both, Phase 1 and Phase 2, no death occurred during the ischemia period. All deaths occurred within the first 24 hours of reperfusion, which is consistent with previous demonstrations that reperfusion is more damaging than ischemia itself ${ }^{2,4,7}$.

The type of rats employed, aged between three and four months, receiving food and water ad libitum, usually have a weight gain of 30 grams per week ${ }^{31}$. The weight gain of all the rats subjected to I/R, when allocated into two groups (with IPC, and without IPC), was respectively $7.86 \mathrm{~g}$ and $5.52 \mathrm{~g}$, with a significant difference between the groups $(\mathrm{p}=0.014)$. Furthermore, the weight gain in each group was significantly lower than that observed in the CTR group $(p<0.001)$, These results demonstrate the suppressive effect of $\mathrm{I} / \mathrm{R}$ on the weight gain, as well as the attenuating effect of IPC on this effect of $I / R$.

Avgerinos et al..$^{32}$ reported a significant decrease in plasma malondialdehyde values in rats subjected to IPC followed by 45 minutes of mesenteric $\mathrm{I} / \mathrm{R}$, compared to the rats without IPC. Likewise, we observed a significant reduction in the malondialdehyde values in the lungs of rats that were subjected to 45 minutes of mesenteric ischemia preceded by IPC and followed by two hours of reperfusion. In the group of animals that were subjected to IPC plus 90 minutes of ischemia, the decrease in the malondialdehyde value did not reach the level of significance.

Tsuboi et al. ${ }^{6}$ demonstrated that the CINC-1 levels in the intestinal mucosa increased considerably in rats subjected to 30 minutes of intestinal ischemia followed by 60 minutes of reperfusion. We observed a significant increase of the CINC-1 level in the lungs of the animals subjected to 45 minutes of intestinal ischemia followed by 2 hours of reperfusion, whereas this increase of the CINC-1 level was attenuated in the animals that underwent IPC. Interestingly, we observed a significant reduction of both the CINC-1 and CINC-2 values in the animals subjected to 90 minutes of ischemia followed by reperfusion; however, increased CINC-1 and CINC-2 values were also observed in the animals subjected to IPC followed by 90 minutes of ischemia. The finding of extremely low CINC-1 and CINC-2 values in the animals that were subjected to 90 minutes of ischemia without IPC was interpreted as a lack of response in the animal, which, after 90 minutes of intestinal ischemia, was unable to produce and release the inflammatory mediators. The animals that underwent IPC before 90 minutes of ischemia also had a theoretically greater capacity to provide a response to injury; these animals released greater amounts of inflammatory mediators and, therefore, had higher CINC-1 and CINC-2 values. This hypothesis seems to be supported by the findings of the Phase 1 study: $50 \%$ mortality in the animals that underwent 90 minutes of intestinal ischemia without IPC versus $15 \%$ mortality in the animals in the IPC-90 group.

It was also observed that the CINC-1 and CINC-2 values were both higher in the animals that were subjected to 45 minutes of mesenteric ischemia than in the animals that were subjected to 90 minutes of ischemia. Initially, this result was unexpected because the animals subjected to longer ischemic times have higher mortality and, therefore, should have higher values of pro inflammatory cytokines. This finding seems to reconfirm the hypothesis that the animals subjected to prolonged mesenteric ischemia (for 90 minutes in this study) are in an advanced stage of the disease and bowel inflammation and, therefore, would be unable to produce an adequate amount of pro-inflammatory cytokines. Conversely, the animals subjected to the same period of ischemia preceded by IPC would be able to produce an adequate amount of these cytokines because of the protective effect of IPC.

Sola et al..$^{30,33}$ observed a decrease in serum malondialdehyde levels caused by the effect of IPC in rats subjected to intestinal I/R. A similar result was observed in the present study, indicated by the reduction of the pulmonary malondialdehyde value in rats subjected to 45 minutes of intestinal ischemia.

As it has been reported, intestinal ischemia also occurs during abdominal surgeries that are not performed directly on the intestine, and also during extra-abdominal surgeries ${ }^{11,25,26}$. However, despite this potentially promising result, further studies are necessary to characterize the possibility of using intestinal IPC in humans. 


\section{Conclusion}

Ischemic preconditioning reduces mortality, inflammatory process and oxidative stress in rats subjected to intestinal ischemia and reperfusion.

\section{References}

1. Wu B, Ootani A, Iwakiri R, Fujise T, Tsunada S, Toda S, Fujimoto K Ischemic preconditioning attenuates ischemia-reperfusion-induced mucosal apoptosis by inhibiting the mitochondria-dependent pathway in rat small intestine Am J Physiol Gastrointest Liver Physiol. 2004;286:G580-7. PMID: 15010362.

2. Huang Y, Shan J, Wang C, Ma J, Li D, Li L, Li S, Li Y. Can ischemic preconditioning alone really protect organs from ischemia reperfusion injury in transplantation. Transplant Immunol. 2009;20:127-31. PMID: 18761408.

3. Aksoyek S, Cinel I, Avlan D, Cinel L, Ozturk C, Gurbuz P, Nayci A, Oral U. Intestinal ischemic preconditioning protects the intestine and reduces bacterial translocation. Shock. 2002;18:476-80. PMID: 12412630

4. Silva Jr OC, Centurion S, Pacheco EG, Brisotti JL, Oliveira AF, Dal Sasso K. Basics aspects of the ischemia reperfusion injury and of the ischemic preconditioning. Acta Cir Bras. 2002;17(Suppl. 3):96100. doi: 10.1590/S0102-86502002000900020.

5. Shibata F, Kato H, Konishi K, Okumura A, Ochiai H, Nakajima $\mathrm{K}$, Al-Mokdad M, Nakagawa H. Differential changes in the concentrations of cytokine-induced neutrophil chemoattractant (CINC)-1 and CINC-2 in exudates during rat lipopolysaccharideinduced inflammation. Cytokine. 1996;8(3):222-6. PMID: 8833037.

6. Tsuboi H, Naito Y, Katada K, Takagi T, Handa O, Kokura S. Role of the thrombin/protease-activated receptor 1 pathway in intestinal ischemia-reperfusion injury in rats. Am J Physiol Gastrointest Liver Physiol. 2007;292:G678-83. 98. PMID: 17023547.

7. Parks DA, Granger DN. Contributions of ischemia and reperfusion to mucosal lesion formation. Am J Physiol. 1986;250(6 Pt 1):G74953. PMID: 3717337.

8. Kozar RA, Holcomb JB, Hassoun HT, Macaitis J, DeSoignie R, Moore FA. Superior mesenteric artery occlusion models shockinduced gut ischemia-reperfusion. J Surg Res. 2004;116:145-50. PMID: 14732361 .

9. Koike K, Moore FA, Moore EE, Poggetti RS, Tuder RM, Banerjee A. Endotoxin after gut ischemia/reperfusion causes irreversible lung injury. J Surg Res. 1992;52(6):656-62. PMID: 1326681.

10. Ito K, Ozasa H, Kojima N, Miura M, Iwa T, Senoo H, Horikawa S. Pharmacological preconditioning protects lung injury induced by intestinal Ischemia/reperfusion in rat. Shock. 2003;19(5):462-8. PMID: 12744491.

11. Jácome DT, Abrahão MS, Morello RJ, Martins JL, Medeiros AC, Montero EFS. Different intervals of ischemic preconditioning on small bowel ischemia-reperfusion injury in rats. Transplant Proc. 2009;41:827-9. PMID: 19376363.

12. Murry CE, Jennings RB, Reimer KA. Preconditioning with ischemia: a delay of lethal cell injury in ischemic myocardium. Circulation. 1986;74(5):1124-36. PMID: 3769170.

13. Hotter G, Closa D, Prados M, Fernandez-Cruz L, Prats N, Gelpi $\mathrm{E}$, Roselló-Catafau J. Intestinal preconditioning is mediated by a transient increase in nitric oxide. Biochem Biophys Res Commun. 1996;222:27-32. PMID: 8630069.

14. Wang SF, Li GW. Early protective effect of ischemic preconditioning on small intestinal graft in rats. World J Gastroenterol. 2003;9:186670. PMID:12918141.
15. Sola A, Rosello-Catafau J, Alfaro V, Pesquero J, Palacios L, Gelpi E, Hotter G. Modification of glyceraldehyde-3phosphate dehydrogenase in response to nitric oxide in intestinal preconditioning. Transplantation. 1999;67:1446-52. PMID: 10385084.

16. Coelho AM, Machado MC, Takahashi HK, Sampietre SN, Stefano JT, Leite AZ, Curi R, D'Albuquerque LA. Ischemic preconditioninglike effect of polyunsaturated fatty acid-rich diet on hepatic ischemia/reperfusion injury. J Gastrointest Surg. 2011;15:1679-88. PMID: 21826546.

17. Neves JS, Abrahão MS, Netto AAS, Montero EFS, Gonzalez AM. Effects of ischemic preconditioning associated to different preservation solutions in protecting the intestinal graft. Acta Cir Bras. 2011;26(5):496-502. PMID: 21952665.

18. McCallion K, Wattanasirichaigoon S, Gardiner KR. Ischemic preconditioning ameliorates ischemia- and reperfusion induced intestinal epithelial hyperpermeability in rats. Shock. 2000;14:42934. PMID: 11049105.

19. Mallick IH, Winslet MC, Seifalian AM. Ischemic preconditioning of small bowel mitigates the late phase of reperfusion injury: heme oxygenase mediates cytoprotection. Am J Surg. 2010;199(2):22331. PMID: 19362701.

20. Tamion F, Richard V, Lacoume Y, Thuillez C. Intestinal preconditioning prevents systemic inflammatory response in hemorrhagic shock. Role of HO-1. Am J Physiol Gastrointest Liver Physiol. 2002;283:G408-4. PMID: 12121889.

21. Tamion F, Richard V, Renet S, Thuillez C. Intestinal preconditioning prevents inflammatory response by modulating heme oxygenase-1 expression in endotoxic shock model. Am J Physiol Gastrointest Liver Physiol. 2007;293:G1308-14. PMID: 17823216.

22. Mallick IH, Yang W, Winslet MC, Seifalian AM. Ischemiareperfusion injury of the intestine and protective strategies against injury. Dig Dis Sci. 2004;49:1359-77. PMID: 15481305.

23. Zitta K, Meybohm P, Bein B, Heinrich C, Renner J, Cremer J, Steinfath M, Scholz J, Albrecht M. Serum from patients undergoing remote ischemic preconditioning protects cultured human intestinal cells from hypoxia-induced damage: involvement of matrixmetalloproteinase-2 and -9. Mol Med. 2012;1(8):29-37. PMID: 22009279.

24. Grotto D, Santa Maria LD, Boeira S, Valentini J, Charão MF, Moro AM, Nascimento PC, Pomblum VJ, Garcia SC. Rapid quantification of malondialdehyde in plasma by high performance liquid chromatography-visible detection. J Pharm Biomed Anal. 2007;43(2):619-24. PMID: 16949242.

25. Rupani B, Caputo FJ, Watkins AC, Vega D, Magnotti LJ, Lu Q, Xu da Z, Deitch EA. Relationship between disruption of the unstirred mucus layer and intestinal restitution in loss of gut barrier function after trauma hemorrhagic shock. Surgery. 2007;141:481-9. PMID: 17383525.

26. Korth $\mathrm{U}^{1}$, Krieter $\mathrm{H}$, Denz C, Janke C, Ellinger K, Bertsch $\mathrm{T}$, Henn $\mathrm{C}$, Klein J.. Intestinal ischaemia during cardiac arrest and resuscitation: comparative analysis of extracellular metabolites by microdialysis. Resuscitation. 2003;58:209-17. PMID: 12909384.

27. Moraes LB, Murakami AH, Fontes B, Poggetti RS, Van Roojen N, Younes RN. Gut ischemia/reperfusion induced acute lung injury is an alveolar macrophage dependent event. J Trauma Inj Inf Crit Care. 2008;64:1196-200. PMID: 18469641.

28. Kubes P, Payne D, Woodman RC. Molecular mechanisms of leukocyte recruitment in postischemic liver microcirculation. Am J Physiol Gastrointest Liver Physiol. 2002;283(1):139-47. doi: 10.1152/ajpgi 00058.2002.

29. Figueira ERR, Bacchella T, Coelho AMM, Sampietre SN, Molan NAT, Leitão RM, Machado MC. Timing-dependent protection 
of hypertonic saline solution administration in experimental liver ischemia/reperfusion injury. Surgery. 2010;147(3):415-23. PMID: 20004454.

30. Sola A, Hotter G, Prats N, Xaus C, Gelpi E, Roselló-Catafau J. Modification of oxidative stress in response to intestinal preconditioning. Transplantation. 2000;69:767-72. PMID: 10755524.

31. Tomanari GY, Pine AS, Silva MTA. Wistar rats under water and food restriction. Rev Bras Ter Comp Cogn. 2003;5(1):57-71.

32. Avgerinos ED, Kostopanagiotou G, Costopanagiotou C, Kopanakis N, Andreadou I, Lekka M. Nakos G, Smyrniotis V. Intestinal preconditioning ameliorates ischemia-reperfusion induced acute lung injury in rats: an experimental study. J Surg Res. 2010;160(2):294-301. PMID: 19439321.

33. Sola A, Alfaro V, Hotter G. Intestinal ischemic preconditioning: Less xanthine accumulation relates with less apoptosis. Apoptosis. 2004;9:353-61. PMID: 15258467.

\section{Correspondence:}

Daniel Faria de Campos Pinheiro

Rua Cubatão, 929/cj. 75

04013-043 São Paulo - SP Brasil

Tel.: (55 11)99998-5512

daniel@campospinheiro.com

Received: Sept 08, 2015

Review: Nov 10, 2015

Accepted: Dec 12, 2015

Conflict of interest: none

Financial source: none

${ }^{1}$ Research performed at Laboratory of Surgical Physiopathology (LIM62), Department of General and Trauma Surgery, Medical School, University of São Paulo (USP), Brazil. Part of PhD degree thesis, Postgraduate Program in Surgical Clinics. Tutor: Belchor Fontes. 